Objectives To evaluate clinicopathological features and survival outcomes of pure dysgerminoma of the ovary

Methods We retrospectively recorded clinicopathological and therapeutic data of 31 patients with pure dysgerminoma of ovary treated at the Salah Azaiez Institute of Tunisia between 1970 and 2012.

Results The median age was 22 years (12-60 years). The distribution of patients according FIGO stage was as follow:stage I:16 (51.6\%),stage II:3 (9.7\%),stage III: 11(35.5\%) and stage IV:1 case.Radical surgery was performed in 11 patients and conservative surgery in 20 patients (64.5\%) associated with node picking lymphadenectomy in 7 cases and complete lymphadenectomy in 7 cases. Complete macroscopic resection was obtained in 22 cases (70.96\%) and lymph node metastasis was observed in $51.1 \%$ of cases. Adjuvant chemotherapy was indicated in 15 cases and adjuvant radiotherapy in 10 cases. After a mean time follow-up of 74 months (7-182 months), complete remission was observed in 26 patients. The 5 -year progression-free survival (PFS) was $85.2 \%$. The 5 -year overall survival (OS) was $89.5 \%$ and was significantly decreased in the advanced stage $(100 \%$ in stage I-II vs $75 \%$ in stage III-IV; $\mathrm{p}=0.02)$. There was a significant difference in OS and PFS between complete resection and residual disease groups (100\% vs $67.5 \% ; \mathrm{p}=0.03$ and 88.9 vs $75 \% ; \mathrm{p}=0.03$, respectively). No difference in OS and PFS was noted following stratification by age $\leq$ or $>15$ years $(\mathrm{p}=0.36$ and $\mathrm{p}=0.1)$, tumor size $\leq$ or $>20 \mathrm{~cm}(\mathrm{p}=0.27$ and $\mathrm{p}=0.68)$ and conservative or radical surgery $(\mathrm{p}=0.87$ and $\mathrm{p}=0.17)$.

Conclusions Macroscopic residual disease, as well as advanced FIGO stage, were the main prognostic factors in pure dysgerminoma of the ovary.

\section{EPV201/\#44 REAL-WORLD DATA ANALYSIS OF SECOND-LINE POLY (ADP-RIBOSE) POLYMERASE INHIBITOR MAINTENANCE THERAPY IN PATIENTS WITH ADVANCED OVARIAN CANCER}

${ }^{1} \mathrm{R}$ Reid*, ${ }^{2} \mathrm{Y} \mathrm{Xie}_{\mathrm{e}}{ }^{2} \mathrm{~J}$ Shi, ${ }^{3} \mathrm{~K}$ Wallace. ' US Oncology, Virginia Cancer Specialists, Medical Oncology, Fairfax, USA; ${ }^{2}$ Ontada, Real World Research, The Woodlands, USA; ${ }^{3}$ Clovis Oncology, Inc., Health Economics and Outcomes Research, Boulder, USA

\subsection{6/ijgC-2021-IGCS.272}

Objectives Poly(ADP-ribose) polymerase inhibitors (PARPis) have been recommended since 2017 as second-line (2L) maintenance treatment by the NCCN for ovarian cancer (OC) patients with or without BRCA1 or BRCA2 (BRCA) alterations. Here, we assessed PARPi use from real-world data.

Methods From the iKnowMed electronic health record database of the US Oncology Network (>470 sites), adult females were included if they were diagnosed with advanced OC, received a $2 \mathrm{~L}$ platinum-containing regimen for advanced OC, and had $\geq 2$ visits between 1 January 2016 and 1 July 2020 . Patients were followed until 31 October 2020, last patient record, or death, whichever occurred earliest. A 24-month landmark survival analysis was performed.

Results Out of 11,494 patients diagnosed with advanced OC, 1051 met the inclusion criteria; 513/1051 (49\%) subsequently received any maintenance therapy (table 1 ). The proportion of patients receiving 2L PARPi maintenance increased from 17\% in 2018 to $34 \%$ in 2019 but decreased to $22 \%$ in 2020 (figure 1). Among $\mathrm{BRCA}^{+}$patients, 33\% (46/140) received 2L PARPi maintenance, while documented $\mathrm{BRCA}^{-}$patients received PARPi maintenance at a significantly lower rate $(23 \%$; $155 /$
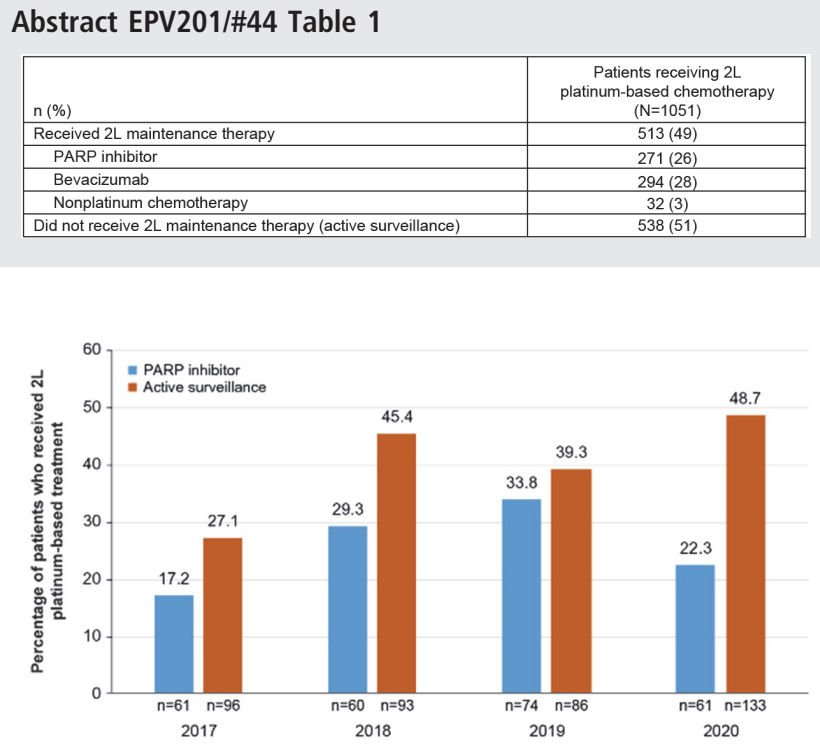

Abstract EPV201/\#44 Figure 1 Propotion of patients receiving 2L PARPi maintenance (active surveillance)

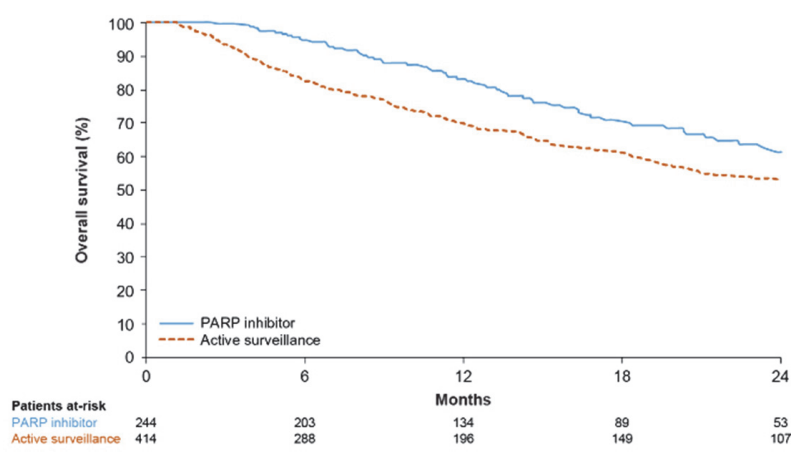

Abstract EPV201/\#44 Figure 2 Overall survival

622; $\mathrm{P}=0.0192$ ). Survival at 24 months was significantly higher with PARPi maintenance vs active surveillance: $61.2 \%$ (95\% CI, 52.4\%-68.8\%) vs 53.0\% (95\% CI, 47.1\%-58.7\%; log-rank $\mathrm{P}=0.0045$ ) (figure 2).

Conclusions Our data suggest a significant proportion of eligible patients are not receiving $2 \mathrm{~L}$ maintenance therapy despite treatment guideline recommendations and apparent survival benefits associated with its use.

\section{EPV202/\#446 SENTINEL LYMPH NODE IDENTIFICATION IN EARLY STAGE OVARIAN CANCER: IS IT STILL POSSIBLE AFTER PRIOR TUMOR RESECTION?}

${ }^{1} \mathrm{P}$ Laven*, ${ }^{2} \mathrm{R}$ Kruitwagen, ${ }^{2} \mathrm{~S}$ Lambrechts, ${ }^{3} \mathrm{~T}$ Van Gorp, ${ }^{4} \mathrm{~B}$ Slangen, ${ }^{5} \mathrm{P}$ Zusterzeel, ${ }^{6} \mathrm{~J}$ Van Der Pol. 'Maastricht Universitair Medisch Centrum, Gynaecology, Maastricht, Netherlands; ${ }^{2}$ Maastricht University Medical Centre, Gynecology, Maastricht, Netherlands; ${ }^{3}$ University Hospital Leuven, Gynaecological Oncology, Leuven, Belgium; ${ }^{4}$ Maastricht University Medical Center, Gynaecology and Obstetrics, Maastricht, Netherlands; ${ }^{5}$ Radboud Univeristy Hospital, Gynaecological Oncology, Nijmegen, Netherlands; ${ }^{6}$ Maastricht Universitair Medisch Centrum, Nuclear Medicine, Maastricht, Netherlands

\subsection{6/ijgc-2021-IGCS.273}

Objectives Sentinel lymph node (SLN) detection in ovarian cancer is feasible when tracers are injected before the pathological ovary is resected. This study aims to investigate 